\title{
Spatial and Temporal Fracture Pattern in Breast and Gynecologic Cancer Survivors
}

\author{
Susanta K Hui ${ }^{1,2}{ }^{\bowtie}$, Luke Arentsen ${ }^{1}$, Anjali Wilcox ${ }^{3}$, Ryan Shanley², Douglas Yee ${ }^{4,2}$, Rahel Ghebre ${ }^{2,3}$ \\ 1. Department of Therapeutic Radiology, University of Minnesota, Minneapolis; \\ 2. Masonic Cancer Center, University of Minnesota, Minneapolis; \\ 3. Department of Obstetrics and Gynecology, University of Minnesota, Minneapolis; \\ 4. Department of Medicine, University of Minnesota, Minneapolis. \\ $\triangle$ Corresponding author: Susanta K Hui, Department of Therapeutic Radiology, University of Minnesota, 420 Delaware Street SE, Mayo Mail Code \\ 494, Minneapolis, MN 55455. Phone: 612-626-4484, Fax: 612-626-7060 E-mail: huixx019@umn.edu.
}

(c) Ivyspring International Publisher. This is an open-access article distributed under the terms of the Creative Commons License (http://creativecommons.org/ licenses/by-nc-nd/3.0/). Reproduction is permitted for personal, noncommercial use, provided that the article is in whole, unmodified, and properly cited.

Received: 2014.08.07; Accepted: 2014.10.06; Published: 2015.01.01

\begin{abstract}
Objective(s): To assess skeletal wide fracture location and time of fracture after cancer treatment Study Design: One hundred thirty-nine women diagnosed with breast or gynecologic cancer between 2003 and 2012 that subsequently had a radiologic diagnosis of fracture were identified retrospectively using electronic medical records. Results were compared with skeletal fracture pattern previously reported for a general population.

Results: Skeletal fractures in cancer patients occur throughout the entire skeleton similar to general population. The most common sites were vertebrae (16\%), feet and toes $(15 \%)$, ribs $(12 \%)$, hands and fingers (10\%), and pelvis (8\%). Fracture incidence was observed starting within the first year of survivorship, and continued to after five years. The median time from cancer diagnosis to fracture varied by age $(p<0.01)$, from a high of 3.2 years for ages $50-59$ to a low of 1.2 years for patients older than 70 .

Conclusion: The pattern of skeletal fracture is similar between cancer survivor and general population. Contrary to general assumption, survivors can experience skeletal fracture early after cancer treatment, especially at an older age. Thus, cancer survivorship care should include assessment of early time points with improved management of cancer treatment related bone injury.
\end{abstract}

Key words: Bone loss, chemotherapy, radiation, quantitative computed tomography.

\section{Introduction}

Women who receive cancer therapy experience an increased fracture risk from treatment-induced bone loss compared to women without cancer associated treatment [1-3]. Recent reports show very high $(36.9 \%)$ incidence of insufficiency fracture within 2 years of treatment [4]. Because of advances in cancer treatment, the number of cancer survivors continues to grow with an estimated 18 million cancer survivors by 2022 at risk for developing treatment related complications [5]. Most knowledge gained on fracture associated outcomes including anatomical location is primarily derived from an aging general population $[6,7]$. These studies found more than $70 \%$ of fractures in women between 40 and 79 years old occurred at locations other than the lumbar spine and hip. Furthermore, in the limited reports of fracture outcomes among cancer survivors, fractures are regarded as late treatment complication. There is a lack of data regarding the time of fracture occurrence in relation to treatment received and if there are certain sites more prone to fracture among cancer survivors compared to fractures experienced due to aging in the general population.

In this retrospective study, our objective is to report relative occurrence of skeletal wide fracture in cancer survivors and to compare the pattern with the 
general population.

\section{Materials and Methods}

Women diagnosed with breast or gynecologic cancer and who subsequently had a radiologic diagnosis of fracture were included. One hundred thirty-nine women treated at the University of Minnesota Medical Center between 2003 and 2012 were identified retrospectively using electronic medical records. The study was approved by the University of Minnesota Institutional Review Board. Data extracted from review of medical records included age, cancer type, date of diagnosis of cancer, treatment received, date and site of fracture. Breast cancer patients underwent multimodality (chemotherapy, hormone therapy, radiation therapy) treatment as defined by their oncologists. The treatment regimen for ovarian cancer patients included standard systemic chemotherapy including paclitaxel and carboplatin. Patients diagnosed with an endometrial cancer were primarily treated using external beam radiation therapy (EBRT) to the pelvis (median dose, 45-50 cGy in 25-28 fractions) and adjuvant chemotherapy.

(A) Percent of fracture by skeletal site

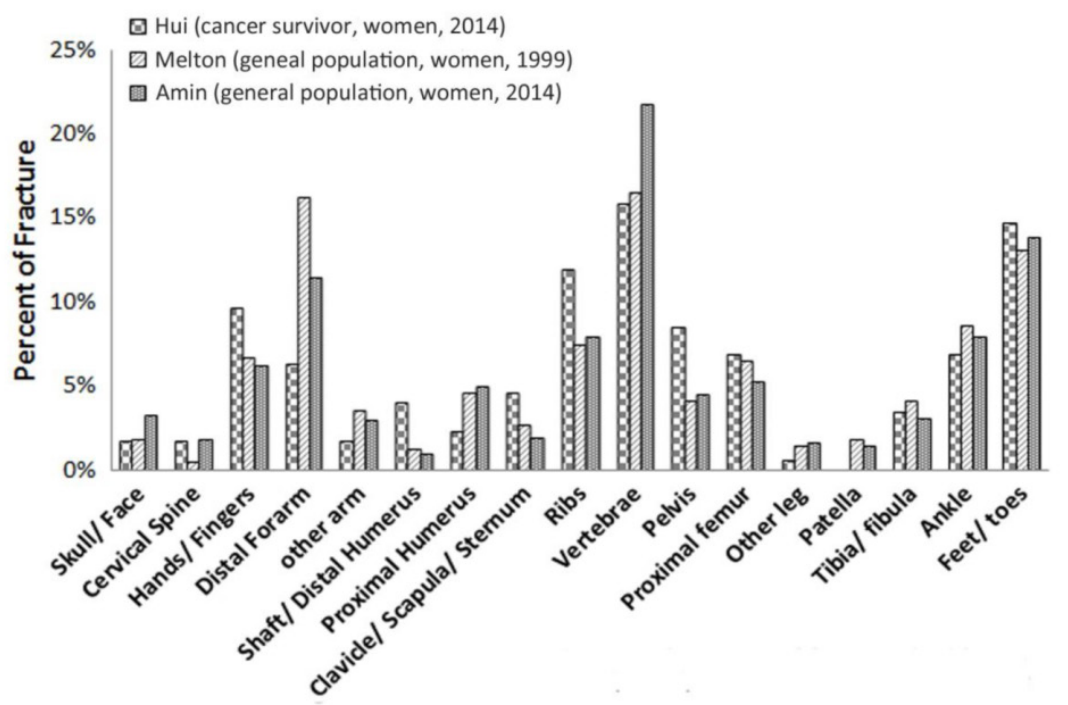

(B) Percent of fracture incidence by age group

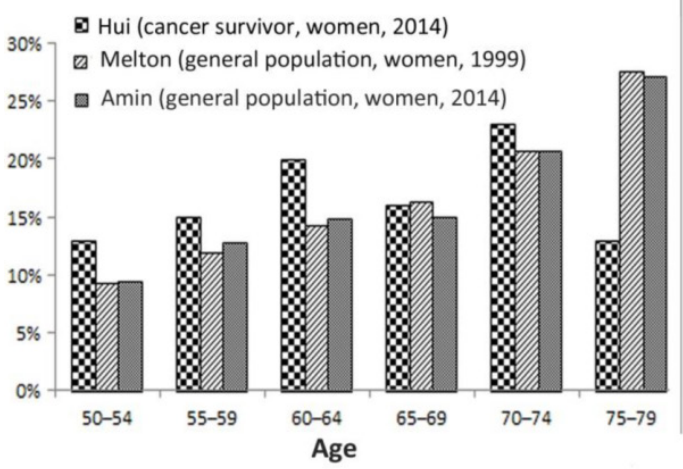

(C) Temporal pattern of fracture incidence in cancer survivors from diagnosis

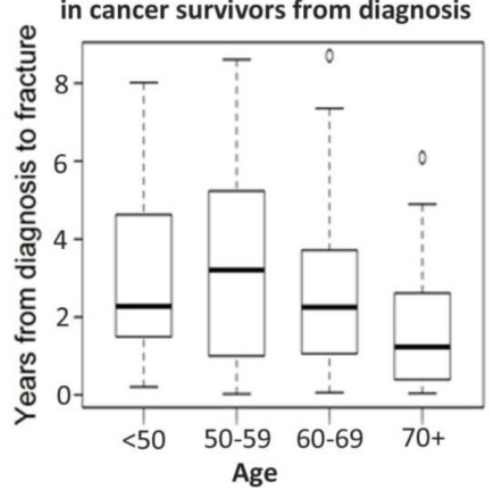

Figure 1. Spatial \& temporal pattern of fracture incidence. A. Comparative evaluation fracture incidence in various skeletal sites between cancer patients and general health population (corrected for age and gender). B. Percent of fractures incidences between six 5-year age groups. Fractures afflicting this study's cancer survivors are compared with fractures to women of similar age in the general population. $C$. Fracture incidence measured by years from the diagnosis of cancer for four different age groups. 
To compare the fracture pattern due to age to the general population, cancer survivors and data from general population studies were separated into six 5 -year age groups (Figure 1B). Figure $1 \mathrm{C}$ shows the temporal fracture pattern for cancer survivors based on years since treatment. Fracture incidence was observed starting within the first year of survivorship, and continued up to five years and longer. Among this cohort, $63 \%$ of fractures were identified within the first 3 years but only $15 \%$ beyond the $5^{\text {th }}$ year. Median time from cancer diagnosis to fracture varied by age $(\mathrm{p}<0.01)$, from a high of 3.2 years for ages $50-59$ to a low of 1.2 years for ages $\geq 70$.

Table 1. Demographic: characteristics of our patient population receiving different treatment. Many patients received combination of treatment.

\begin{tabular}{llllll}
\hline Cancer site & $\mathrm{N}$ & $\begin{array}{l}\text { Age } \\
\text { median } \\
\text { (range) }\end{array}$ & $\begin{array}{l}\text { Chemotherapy } \\
\mathrm{N}(\%)\end{array}$ & $\begin{array}{l}\text { Radiation } \\
\mathrm{N}(\%)\end{array}$ & $\begin{array}{l}\text { Hormonal } \\
\text { treatment } \\
\mathrm{N}(\%)\end{array}$ \\
\hline Breast & 87 & $61(24-95)$ & $43(49 \%)$ & $58(67 \%)$ & $57(66 \%)$ \\
Endometrium & 21 & $55(49-83)$ & $9(43 \%)$ & $16(76 \%)$ & $1(5 \%)$ \\
Ovary & 15 & $63(34-90)$ & $14(93 \%)$ & $3(20 \%)$ & $1(7 \%)$ \\
Other & 16 & $49(25-91)$ & $13(81 \%)$ & $11(69 \%)$ & $3(19 \%)$ \\
Total & 139 & $62(24-95)$ & $79(56 \%)$ & $88(63 \%)$ & $62(45 \%)$ \\
\hline
\end{tabular}

\section{Discussion}

This pilot study shows skleteal wide fractures among breast and gynecologic cancer survivors. The wide distrubution of antatomical fracture locations is not soley linked to site of treatment, although the direct radiation injury can not be isolated within this study. Post treatment time of fracture incidence appears to be very early, within years.

Fracture in general population indicates a skeletal wide event rather than a few common sites such as spine and hip [6, 7]. Our study is the first to reveal that fracture pattern in cancer survivors may have similar fracure pattern as in general population. Pelvic radiation associated side effect of pelvic fracture is documented [2]. Our study is the first report with a comprehensive skeletal fracture distribution report. Based on our report the sites of potential fracture occurences among cancer survivors extends beyond the spefic radiation bone injury site. Specifically, major fracture sites, including vertebra and pelvis, accounted for only $25 \%$ of fracture events. There is a large unaccounted fracture occurence outside of the commonly screened sites utilzing standard methods. Among cancer survivors, consideration for bone health assessment should take into account the wide distribution of potential fractures beyond standard bone screening locations.
There were also differences seen in the age distribution of cancer survivors at the time of fracture compared to studies of the general population. Both previously mentioned studies show that as indivdiuals age, the number of fractures also increases $[6,7]$. Another study found that fracture incidence increases exponentially with incrased age and decreasing radius bone mass [8]. However, for cancer survivors, this characteristic of increased fracture with age is disrupted (Figure 1B). Cancer related mortality could effect the pattern, which was not investigated in the present study. In future, with larger number of subjects will provide more accurate temporal (post treatment and age related) distribution of fracture incidence.

In report by Blaes et al., post treatment pathologic fracture among soft-tissue sarcoma patients was identified to increase with time with overall incidence reported at $9 \%$ at 10 years [9]. In a study of gynecologic cancer patientes treated with radiation to the pelvis, the fracture incidence within the radiation field was $11 \%$ at 5 years and $15 \%$ at 10 years [3]. Early occurrences of fracture observed among our patient population suggests a need for early bone health follow up. Poor bone health (osteoporosis or osteopenia) could accelerate fracture events. The onset of early fractures may also be associated with underlying bone health status proceeding cancer therapy. Older age, especially above age 70, could influence accelerated fracture after treatment. However, it has never been clinical practice to assess bone health prior to treatment. We were not able to discern the cause of fracture. Early fracture after treatment diagnosis may represent increased frailty induced by the treatment itself [10].

Though there are limitations in this retrospective study (e.g. small sample size, does not include all cancers, women only), our study reveals that cancer treatment may cause early and skeletal wide fracture. Further study is underway to extend current analysis to all cancers, male and female. The standard assessment for osteoporosis, an indicator of fracture risk, is by Dual-energy x-ray absorptiometry (DEXA) scan. Although clear guidelines exist regarding its role and interpretation within the general population, no clear recommendations exists among cancer survivors. Specifically, there are no recommended time for bone health screening after cancer treatment outside of the general recommendations for an aging population.

We previously demonstrated that routine CT scans frequently used for cancer surveillance could also assess volumetric bone mineral density of cancellous bone [11]. Having the ability to measure bone density as part of the routine cancer follow up of pa- 
tients with no added cost, time or radiation dose will allow for the prospective evaluation of bone mineral changes. Furthermore, cytotoxic cancer treatments accelerate marrow adipogenesis [12], a potential factor for bone damage. New CT imaging techniques have been developed with an ability to measure rapid bone and marrow damage and assess comprehensive treatment management for cancer survivors [13]. Due to the increasing number of young cancer survivors, addressing bone health post treatment is of clinical signficance.

\section{Acknowledgement}

This work is supported by the National Institute of Arthritis and Musculoskeletal and Skin Diseases (NIAMS) (1RO3-AR05333-01), PHS Cancer Center (P30 CA77598). We acknowledge James R Essler for helping to identify records from electronic medical records.

\section{Conflict of interest}

Authors do not have any conflict of interest.

\section{References}

1. Chen Z, Maricic M, Bassford T, Pettinger M, Ritenbaugh C, Lopez A, et al. Fracture risk among breast cancer survivors: Results from the Women's Health Initiative Observational Study. Archives of Internal Medicine. 2005; 165: 552.

2. Baxter N, Habermann E, Tepper J, Durham S, Virnig B. Risk of pelvic fractures in older women following pelvic irradiation. JAMA. 2005; 294: 2587.

3. Grigsby PW, Roberts HL, Perez CA. Femoral neck fracture following groin irradiation. International Journal of Radiation Oncology* Biology* Physics. 1995; 32: 63-7.

4. Tokumaru S, Toita T, Oguchi M, Ohno T, Kato S, Niibe Y, et al. Insufficiency fractures after pelvic radiation therapy for uterine cervical cancer: an analysis of subjects in a prospective multi-institutional trial, and cooperative study of the Japan Radiation Oncology Group (JAROG) and Japanese Radiation Oncology Study Group (JROSG). International journal of radiation oncology, biology, physics. 2012; 84: e195-200. doi:10.1016/j.ijrobp.2012.03.042.

5. de Moor JS, Mariotto AB, Parry C, Alfano CM, Padgett L, Kent EE, et al. Cancer survivors in the United States: prevalence across the survivorship trajectory and implications for care. Cancer Epidemiology Biomarkers \& Prevention. 2013; 22: 561-70.

6. Amin S, Achenbach SJ, Atkinson EJ, Khosla S, Melton LJ. Trends in Fracture Incidence: A Population-Based Study Over 20 Years. Journal of Bone and Mineral Research. 2014; 29: 581-9.

7. Melton III LJ, Crowson C, O'Fallon W. Fracture incidence in Olmsted County, Minnesota: comparison of urban with rural rates and changes in urban rates over time. Osteoporosis International. 1999; 9: 29-37.

8. Hui SL, Slemenda CW, Johnston Jr CC. Age and bone mass as predictors of fracture in a prospective study. Journal of clinical investigation. 1988; 81: 1804.

9. Blaes AH, Lindgren B, Mulrooney DA, Willson L, Cho LC. Pathologic femur fractures after limb-sparing treatment of soft-tissue sarcomas. Journal of Cancer Survivorship. 2010; 4: 399-404.

10. Bennett JA, Winters-Stone KM, Dobek J, Nail LM. Frailty in older breast cancer survivors: age, prevalence, and associated factors. Oncology nursing forum: Onc Nurs Society; 2013. p. E126-E34.

11. Hui S, Khalil A, Zhang Y, Coghill K, Le C, Dusenbery K, et al. Longitudinal assessment of bone loss from diagnostic computed tomography scans in gynecologic cancer patients treated with chemotherapy and radiation. American Journal of Obstetrics and Gynecology. 2010.

12. Bolan PJ, Arentsen L, Sueblinvong T, Zhang Y, Moeller S, Carter JS, et al. Water-fat MRI for assessing changes in bone marrow composition due to radiation and chemotherapy in gynecologic cancer patients. Journal of Magnetic Resonance Imaging. 2013; 38: 7.

13. Hui Susanta K, Brown Keenan, Grammens Gary, Ghebre Rahel G, Haddad Tufia, Minenko Anne G, et al. Development of DEQCT to monitor bone and bone marrow composition in cancer survivors. JOURNAL OF WOMEN'S HEALTH. 2010; 19: 1786. 